\title{
Induction of BALT in the absence of IL-17
}

\section{To the Editor:}

We read with interest the paper by RangelMoreno et al. published in the July 2011 issue of Nature Immunology $y^{1}$. The authors applied a mouse model that includes the repeated sensitization of the airways of newborn mice with the Gram-negative bacterial cell-wall component lipopolysaccharide (LPS), which finally leads to the formation of induced bronchus-associated lymphoid tissue (iBALT). On the basis of their findings, the authors postulate that the formation of iBALT depends on the cytokine interleukin 17 (IL-17) and that this finding establishes a new paradigm for how lymphoid follicles form in nonlymphoid organs ${ }^{1}$.

It has been shown that the intranasal or intratracheal application of pathogens such as mouse $\gamma$-herpes virus 68, Mycobacterium tuberculosis or influenza virus triggers massive induction of BALT in adult mice ${ }^{2-5}$. Vaccinia virus is known to efficiently infect organisms via the respiratory route ${ }^{6}$. A single intranasal or intratracheal application of the replicationdeficient poxvirus modified vaccinia virus ankara (MVA) initiates the rapid induction of BALT in mice that not only allows the initiation of anti-MVA adaptive immune responses but also serves as a general site for priming against unrelated antigens present in the lung ${ }^{7}$.

However, when testing a role for IL-17 in the MVA-induced formation of BALT, we did not detect any difference between wild-type mice and mice with targeted deletion of the entire locus encoding IL-17A and IL-17F (Il17 $a^{-/-} I l 17 f^{-/-}$mice; unpublished data). At $12 \mathrm{~d}$ after intranasal application of $1 \times 10^{7}$ international units of MVA, we failed to observe any significant differences between wild-type and $I l 17 a^{-/-} I l 17 f^{-/-}$mice in the number of iBALT structures per lung section, the average size of individual iBALT structures or the total area of all iBALT structures per lung section (Fig. 1a,b). Furthermore, mice of these genotypes were also indistinguishable in the number of $\mathrm{B}$ cell follicles present per lung section and the average size of the follicles, as well as the total follicular area per lung section (Fig. 1c). Furthermore, in mice of both genotypes, most iBALT structures had segregated B cell follicles (wild-type, $65 \% \pm$ $12 \% ; I l 17 a^{-/-} I l 17 f^{-/-}, 61 \% \pm 12 \%$ (mean \pm s.d.)). For the LPS-sensitization model, Rangel-Moreno et al. postulate a role for IL-17 in the induction of the chemokine CXCL13, which they suggest to be important for follicle formation ${ }^{1}$. Although we agree with the view that CXCL13 is important for follicle formation, we did not observe any effect of the absence of IL-17A and IL-17F on the induction of CXCL13 (Fig. 1d), which also helps to explain why we could not find any difference between wild-type
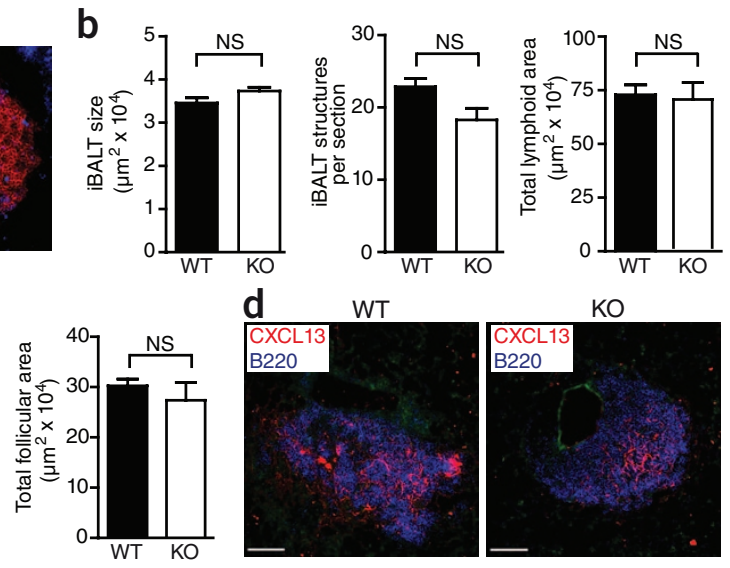

Figure 1 The development of MVA-induced BALT does not require IL-17. (a) Immunohistology of iBALT in the lungs of 10- to 12-week-old C57BL/6 wild-type mice (WT) or C57BL/6 //17a ${ }^{-1-} / / 17 f^{-1}$ mice (KO) antibody to B220 (RA3-3A1), to identify B cell follicles, and to CD3 (17A2), to identify T cell zones

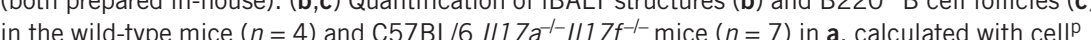
software (Olympus) as the sum of all BALT structures or all follicles per lung section, respectively. NS, C57BL/6 wild-type and $/ / 17 a^{-1-} / / 17 f^{-1-}$ mice infected with MVA as in a, stained with affinity-purified goat antibody to mouse CXCL13 (AF 470; R\&D Systems) and monoclonal antibody to B220. Scale bars (a,d), $100 \mu \mathrm{m}$. Data are representative two independent experiments with four wild-type mice and seven $/ / 17 a^{-1-} / 117 f^{-1-}$ mice $(\mathbf{a}, \mathbf{d})$ or are from two independent experiments with four representative lung sections per mouse (b,c; mean and s.d.).

and $I l 17 a^{-/-} I l 17 f^{-/-}$mice in follicle formation, as described above.

In summary, our data show that BALT can be efficiently induced in the complete absence of IL-17A and its homolog IL-17F. Therefore, we believe that the general conclusion reached by Rangel-Moreno et al. that iBALT formation depends on IL-17 (ref. 1) is inappropriate. The function of IL-17 in the induction of BALT needs clarification for understanding of the role of this cytokine in the biology of ectopic lymphoid structures.

Henrike Fleige ${ }^{1}$, Jan D Haas ${ }^{1}$, Felix R Stahl ${ }^{1}$, Stefanie Willenzon ${ }^{1}$, Immo Prinz ${ }^{1,2}$ \& Reinhold Förster ${ }^{1,2}$

${ }^{1}$ Institute of Immunology, Hannover Medical School, Hannover, Germany.

${ }^{2}$ These authors contributed equally to this work.

e-mail: foerster.reinhold@mh-hannover.de

\section{COMPETING FINANCIAL INTERESTS}

The authors declare no competing financial interests.

1. Rangel-Moreno, J. et al. Nat. Immunol. 12, 639-646 (2011).

2. GeurtsvanKessel, C.H. et al. J. Exp. Med. 206, 2339-2349 (2009).

3. Kahnert, A. et al. J. Infect. Dis. 195, 46-54 (2007).

4. Kocks, J.R. et al. J. Immunol. 182, 6861-6869 (2009).

5. Moyron-Quiroz, J.E. et al. Nat. Med. 10, 927-934 (2004).

6. Reading, P.C. \& Smith, G.L. J. Gen. Virol. 84, 1973-1983 (2003),

7. Halle, S. et al. J. Exp. Med. 206, 2593-2601 (2009). 